\title{
Erratum to: Measuring Callous Unemotional Behaviors in Early Childhood: Factor Structure and the Prediction of Stable Aggression in Middle Childhood
}

\author{
Michael T. Willoughby • W. Roger Mills-Koonce • \\ Nisha C. Gottfredson • Nicholas J. Wagner
}

Published online: 18 October 2013

(C) Springer Science+Business Media New York 2013

\section{Erratum to: J Psychopathol Behav Assess \\ DOI 10.1007/s10862-013-9379-9}

Soon after the online publication of our manuscript (Willoughby, Mills-Koonce, Gottfredson, and Wagner 2013), we determined that a data management error had occurred in the creation of the analysis dataset. This error resulted in some observations being duplicated and others being omitted. Below, we provide corrected study results and conclusions.

\section{Descriptive Statistics}

Updated demographic and behavioral characteristics of the corrected sample are summarized in Table 1. Although the total sample size was unchanged $(N=1,081)$, there were slight changes to the numbers of children characterized as having organized versus disorganized attachments (now Ns $=918$ and 163 , respectively). Only minor and trivial changes were evident with respect to the demographic characteristics of the sample.

The online version of the original article can be found at http://dx.doi.org/ 10.1007/s10862-013-9379-9.

M. T. Willoughby $(\bowtie) \cdot$ N. C. Gottfredson

FPG Child Development Institute, University of North Carolina at Chapel Hill, CB \#8185, Chapel Hill, NC 27599-8185, USA

e-mail: willoughby@unc.edu

W. R. Mills-Koonce $\cdot$ N. J. Wagner

Center for Developmental Science, University of North Carolina at Chapel Hill, Chapel Hill, NC, USA
Moreover, as before, there was no evidence that children with organized versus disorganized attachment status differed meaningfully on any of the demographic variables considered. A minor change in the updated Table 1 is that we now report mean (not sum) scores for ADHD, CU, and ODD.

The corrected bivariate correlations for all study variables, including the time specific indicators of teacher-rated aggression which were used to create the aggression trajectory groups, are summarized in Table 2. Two changes were evident. First, child gender and race, caregiver education, and household income to needs ratio were all more strongly associated with membership in the stable elevated aggression trajectory $(|r| \mathrm{s}=.25-.47)$ than was the case previously. Second, parent-rated ADHD, ODD, and CU behaviors also appeared to be more strongly related to membership in the stable elevated aggression trajectory class $(r \mathrm{~s}=.18-.27)$ than was previously the case. Child disorganization status continued to be unrelated to membership in the stable trajectory class.

\section{Dimensionality of ASEBA Items}

The first objective of this study was to examine the factor structure of 17 parent-reported items from the ASEBA that represented $\mathrm{ADHD}, \mathrm{ODD}$, and $\mathrm{CU}$ behaviors using a large sample of 3-year olds. All of the confirmatory factor models were re-estimated using corrected data. The updated results are summarized in Table 3. Only trivial differences in model fit statistics were evident and none of the substantive conclusions changed. Although the 3 -factor model provided a statistically better fit to the data than did any of the 1- or 
Table 1 Descriptive statistics

$M$ - Mean, $S D$ - Standard Deviation; Superscript A: $N=1011$; Superscript B: $N=1026$; Superscript C: $N=862$; Superscript D: $N=864 ;$ Superscript E: $N=149$; Superscript F: $N=162$

\begin{tabular}{|c|c|c|c|}
\hline & $\begin{array}{l}\text { Total sample } \\
(N=1081)\end{array}$ & $\begin{array}{l}\text { Organized attachment } \\
(N=918)\end{array}$ & $\begin{array}{l}\text { Disorganized attachment } \\
(N=163)\end{array}$ \\
\hline & $\mathrm{M}(\mathrm{SD})$ & $\mathrm{M}(\mathrm{SD})$ & $\mathrm{M}(\mathrm{SD})$ \\
\hline Mother education (Years) & $14.4(2.5)$ & $14.5(2.4)$ & $14.1(2.6)$ \\
\hline Income-to-need & $3.0(2.6)^{\mathrm{A}}$ & $3.0(2.6)^{\mathrm{C}}$ & $3.1(2.9)^{\mathrm{E}}$ \\
\hline ADHD & $0.9(0.4)^{\mathrm{B}}$ & $0.9(0.4)^{\mathrm{D}}$ & $1.0(0.4)^{\mathrm{F}}$ \\
\hline $\mathrm{CU}$ & $0.4(0.3)^{\mathrm{B}}$ & $0.3(0.3)^{\mathrm{D}}$ & $0.4(0.4)^{\mathrm{F}}$ \\
\hline \multirow{2}{*}{ ODD } & $0.7(0.4)^{\mathrm{B}}$ & $0.7(0.4)^{\mathrm{D}}$ & $0.7(0.4)^{\mathrm{F}}$ \\
\hline & $\%$ & $\%$ & $\%$ \\
\hline Child race (White) & 82 & 83 & 75 \\
\hline Child race (Black) & 11 & 10 & 19 \\
\hline Child race (Other) & 7 & 7 & 6 \\
\hline Child gender (Male) & 51 & 52 & 41 \\
\hline Attachment (Disorganized) & 15 & 0 & 100 \\
\hline Stable aggression & 7 & 7 & 10 \\
\hline
\end{tabular}

2-factor models, none of the five initial models met conventional standards for good model fit. However, as before, the revised 3-factor model that introduced four correlated residuals fit the data well (see Table 3). Standardized factor loadings and latent/residual correlations from the final model appear in Fig. 1 (two of the latent and residual correlations had slightly different values using updated data). Chronbach's alpha and maximal reliability estimates for ADHD, ODD, and CU were also unchanged.

\section{Prediction of Stable High Aggression}

The second major objective of this study was to test whether individual differences in parent-reported ADHD, ODD, and CU behaviors, as well as children's disorganization status, at age 3 years were prospectively associated with children's membership in a stable elevated trajectory class that was defined by repeated measures of teacher-rated aggression from grades $1-6$. The same sequence of logistic regression

Table 2 Bivariate correlations between predictors and outcomes

\begin{tabular}{|c|c|c|c|c|c|c|c|c|c|c|c|c|c|c|c|}
\hline & 1 & 2 & 3 & 4 & 5 & 6 & 7 & 8 & 9 & 10 & 11 & 12 & 13 & 14 & 15 \\
\hline \multicolumn{16}{|l|}{1 Male } \\
\hline 2 Black & .04 & & & & & & & & & & & & & & \\
\hline 3 Other Race & -.04 & $-.43 *$ & & & & & & & & & & & & & \\
\hline 4 Mom Education & -.05 & $-.39 *$ & -.11 & & & & & & & & & & & & \\
\hline 5 Income/Needs & -.05 & $-.54 *$ & -.08 & $.42 *$ & & & & & & & & & & & \\
\hline 6 Disorganized & $-.16^{*}$ & $.23^{*}$ & -.07 & -.09 & .02 & & & & & & & & & & \\
\hline 7 ADHD & $.08^{*}$ & $.16^{*}$ & .02 & $-.21 *$ & $-.15^{*}$ & .09 & & & & & & & & & \\
\hline $8 \mathrm{CU}$ & .05 & .09 & .00 & $-.24 *$ & $-.09 *$ & $.10^{*}$ & $.53 *$ & & & & & & & & \\
\hline 9 ODD & .00 & -.02 & .00 & $-.11 *$ & $-.08^{*}$ & .05 & $.57^{*}$ & $.52 *$ & & & & & & & \\
\hline $10 \mathrm{High} /$ Stable Agg & $.25^{*}$ & $.47 *$ & -.08 & $-.33 *$ & $-.35^{*}$ & .11 & $.23 *$ & $.27^{*}$ & $.18^{*}$ & & & & & & \\
\hline 11 Teacher Agg G1 & $.22 *$ & $.22 *$ & -.02 & $-.12 *$ & $-.09 *$ & .07 & $.08^{*}$ & $.10^{*}$ & $.12 *$ & $.46^{*}$ & & & & & \\
\hline 12 Teacher Agg G2 & $.21^{*}$ & $.23^{*}$ & -.02 & $-.14 *$ & -.07 & $.11^{*}$ & $.18^{*}$ & $.16^{*}$ & $.14^{*}$ & $.58^{*}$ & $.43^{*}$ & & & & \\
\hline 13 Teacher Agg G3 & $.15^{*}$ & $.28^{*}$ & -.01 & $-.22 *$ & $-.14 *$ & .06 & $.15^{*}$ & $.19^{*}$ & $.11^{*}$ & $.67 *$ & $.33^{*}$ & $.48^{*}$ & & & \\
\hline 14 Teacher Agg G4 & $.18^{*}$ & $.29^{*}$ & .00 & $-.16^{*}$ & $-.14 *$ & .06 & $.16^{*}$ & $.17^{*}$ & $15^{*}$ & $.60^{*}$ & $.41^{*}$ & $.47^{*}$ & $.49^{*}$ & & \\
\hline 15 Teacher Agg G5 & $.16^{*}$ & $.28^{*}$ & -.11 & $-.17 *$ & $-.12 *$ & .08 & $.11 *$ & $.13^{*}$ & .05 & $.51^{*}$ & $.34 *$ & $.40^{*}$ & $.43 *$ & $.49^{*}$ & \\
\hline 16 Teacher Agg G6 & $.28 *$ & $.21^{*}$ & -.04 & $-.17 *$ & $-.14 *$ & $.09 *$ & $.07 *$ & .06 & .06 & $.53^{*}$ & $.42^{*}$ & $.35^{*}$ & $.39^{*}$ & $.35^{*}$ & $.43^{*}$ \\
\hline
\end{tabular}

Total $N=1,081$; Correlations computed using the WLSMV estimator; $* P<.05$ 
Table 3 Synopsis of confirmatory factor models of ASEBA items
$N=1176$; chi square difference tests computed using DIFTEST option in Mplus; prob - probability; CFI - Comparative Fit Index; RMSEA - Root mean squared error of approximation; $C I$ - Confidence interval

\begin{tabular}{llllll}
\hline Model & Description & $\mathrm{X}^{2}(\mathrm{df})$ & prob & CFI & RMSEA (90 \% CI) \\
\hline 1 & 1 factor & $1402.42(119)$ & $<.001$ & .88 & $.10(.09, .10)$ \\
2 & 2 factor (CU vs. other) & $1300.02(118)$ & $<.001$ & .89 & $.09(.09, .10)$ \\
3 & 2 factor (ODD vs. other) & $1090.86(118)$ & $<.001$ & .91 & $.08(.08, .09)$ \\
4 & 2 factor (ADHD vs. other) & $1041.64(118)$ & $<.001$ & .91 & $.08(.08, .09)$ \\
5 & 3 factor (CU, ODD, ADHD) & $922.09(116)$ & $<.001$ & .92 & $.08(.07, .08)$ \\
& Model 5 vs. Model 1 & $285.06(3)$ & $<.001$ & & \\
& Model 5 vs. Model 2 & $204.96(2)$ & $<.001$ & & \\
& Model 5 vs. Model 3 & $110.23(2)$ & $<.001$ & & \\
& Model 5 vs. Model 4 & $82.25(2)$ & $<.001$ & & \\
& Correlated residuals & $443.02(112)$ & $<.001$ & .97 & $.05(.05, .06)$ \\
\hline
\end{tabular}

models was estimated. The initial covariates only model was now predictive of membership in the stable elevated aggression group, $\chi_{(5)}^{2}=56.5, p<.0001$. Child race and gender, as well as maternal education made independent contributions (see Table 4). In the second model, none of the interactions were statistically significant (see full model of Table 4). Moreover in follow-up models that are not presented, none of the lower order interactions were statistically significant either. The final trimmed model retained all predictors as main effects. The demographic factors continued to exert unique effects. African American (odds ratio and $95 \%$ confidence intervals $(\mathrm{OR}[95 \% \mathrm{CI}])=3.5[1.8-7.0], p=.0003)$ and male children $(\mathrm{OR}[95 \% \mathrm{CI}]=2.0[1.1-3.6], p=.02)$ were all at increased risk for being in the stable elevated aggression trajectory group. Higher levels of maternal educational attainment were associated with a decreased risk for child membership in the stable elevated aggression trajectory group (OR [95\% $\mathrm{CI}]=0.9[0.7-0.98], p=.03)$. Finally, among the behavioral predictors, only CU exerted a unique effect. Specifically, higher levels of maternal ratings of $\mathrm{CU}$ behavior at age 3 were associated with increased risk for membership in the stable elevated aggression trajectory group $(\mathrm{OR}[95 \% \mathrm{CI}]=2.9[1.1-$ 7.7], $p=.03)$. Neither ADHD nor ODD had unique effects (see Table 4).

\section{Conclusions}

The previous conclusions regarding the factor structure of ASEBA items were unchanged. Parents of preschool-aged children can differentiate CU from more commonly assessed dimensions of disruptive behavior. The previous conclusions

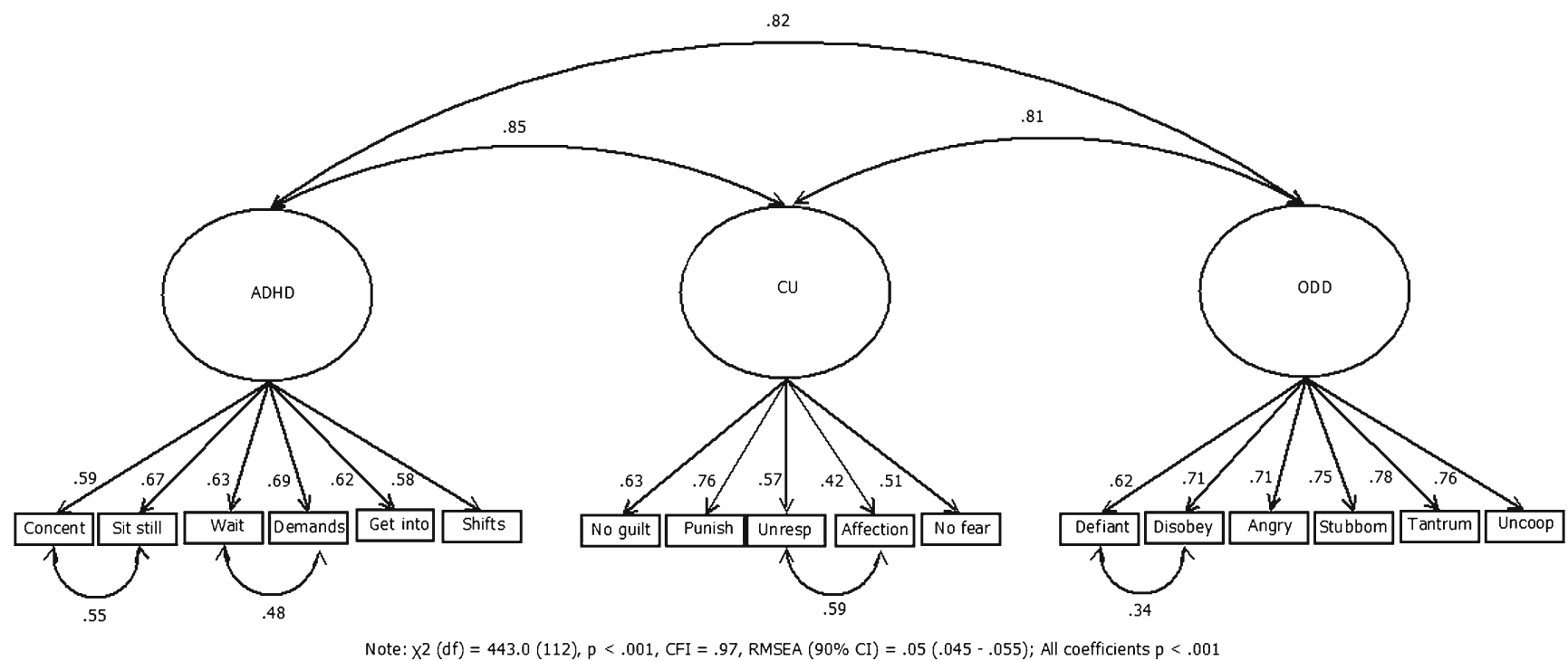

Fig. 1 Standardized coefficients from final confirmatory factor analysis 
Table 4 Synopsis of logistic regression models

\begin{tabular}{|c|c|c|c|c|c|c|c|c|c|}
\hline & \multicolumn{3}{|c|}{ Covariates only } & \multicolumn{3}{|c|}{ Full model } & \multicolumn{3}{|c|}{ Trimmed model } \\
\hline & Beta & SE & $p$ & Beta & SE & $p$ & Beta & SE & $p$ \\
\hline Intercept & -.29 & .87 & .74 & -2.32 & 1.51 & .12 & -1.93 & 1.04 & .06 \\
\hline Black & 1.34 & .32 & $<.001$ & 1.39 & .35 & $<.001$ & 1.27 & .35 & $<.001$ \\
\hline Other Race & .17 & .55 & .76 & .34 & .61 & .58 & .37 & .57 & .51 \\
\hline Male & .75 & .28 & $<.01$ & .71 & .31 & .02 & .69 & .30 & .02 \\
\hline Mother Education & -.21 & .07 & $<.01$ & -.15 & .07 & .03 & -.16 & .07 & .03 \\
\hline Income-to-Needs & -.10 & .09 & .24 & -.10 & .09 & .27 & -.09 & .09 & .29 \\
\hline Disorganized Attachment & & & & .4 .02 & 4.01 & .32 & .34 & .35 & .33 \\
\hline ADHD Sx & & & & -.25 & 1.40 & .86 & .25 & .44 & .57 \\
\hline CU Sx & & & & 5.12 & 2.88 & .08 & 1.06 & .50 & .03 \\
\hline ODD Sx & & & & .15 & 1.85 & .94 & .24 & .45 & .59 \\
\hline ADHD*ODD & & & & .93 & 1.58 & .56 & & & \\
\hline $\mathrm{CU} * \mathrm{ODD}$ & & & & -3.58 & 3.34 & .28 & & & \\
\hline $\mathrm{ADHD} * \mathrm{CU}$ & & & & -1.74 & 2.73 & .52 & & & \\
\hline $\mathrm{ADHD} * \mathrm{CU} * \mathrm{ODD}$ & & & & 1.23 & 2.47 & .62 & & & \\
\hline Disorganizred*ADHD & & & & 4.05 & 4.01 & .31 & & & \\
\hline Disorganized*CU & & & & 4.17 & 7.75 & .59 & & & \\
\hline Disorganized*ODD & & & & 6.74 & 4.99 & .18 & & & \\
\hline Disorganized*ADHD*ODD & & & & -5.38 & 4.48 & .23 & & & \\
\hline Disorganized*CU*ODD & & & & -5.59 & 7.71 & .47 & & & \\
\hline Disorganized*ADHD*CU & & & & -5.12 & 6.54 & .43 & & & \\
\hline Disorganized*ADHD*CU*ODD & & & & 5.36 & 5.44 & .32 & & & \\
\hline$-2 \mathrm{LL}$ & & 482.5 & & & 444.2 & & & 444.21 & \\
\hline Max Rescaled $\mathrm{R}^{2}$ & & .14 & & & .19 & & & .17 & \\
\hline Likelihood Ratio $\chi^{2}$ (df) & & $56.2(5)$ & $<.001$ & & $69.9(20)$ & $<.001$ & & $61.6(9)$ & $<.001$ \\
\hline
\end{tabular}

$N=966 ; S E$ - Standard error; $p$ - Probability; $L L$ - Log likelihood; $S x$ - Symptom

regarding the predictive validity of parent-reported $\mathrm{CU}$ did change. Individual differences in CU behaviors at age 3 were uniquely predictive of stable elevated aggression from 1st-6th grade. Whereas individual differences in ADHD and ODD behaviors at age 3 may be developmentally normative and not predictive of future risk for aggression, individual differences in $\mathrm{CU}$ at age 3 are not normative and may be a risk factor for subsequent aggression. Collectively, these results provide support for the measurement of $\mathrm{CU}$ behaviors in early childhood. 